\title{
El desarrollo de software en Colombia: una perspectiva jurídica
} Software Development in Colombia: A Legal Approach

Submitted: 15/06/2018

Revised: $13 / 07 / 2018$

Accepted: 25/08/2018

\author{
Lainiver Mendoza Munar*
}

\begin{abstract}
Purpose - In recent years, Colombia has been a very active country in the region, supporting the innovation and technology sector. The Ministry of Information and Communication Technologies (MINTIC) has implemented various programs, plans and strategies that from the national government seek to improve innovation and technology, within innovation and technology is the development of software, which as a construction of the human intellect deal with the need to be protected by the intellectual property regulations, specifically by the copyright laws for the Colombian case. Plans such as Live Digital in Colombia address the need of the country to be at the forefront of technology and seek the inclusion of society not only in the management of information technologies, but also in the creation, construction and development of these technologies, which promotes several public policies such as employment generation and poverty reduction. This describes the state of the art of the existing software legislation in Colombia.

Methodology - It is important to determine, within the Colombian legal system, the relevant regulations that regulate intellectual property in relation to the industry that develops software, for which purpose this research is carried out, followed by the study of the software development industry in Colombia, and the identification of regulations that exist on the matter.
\end{abstract}

Findings - It was possible to condense the state of the art in the legal field of software within the category of Copyrights in Colombia conceived, from the legal perspective, as a set of products such as computer program, the program description and the auxiliary material.

Practical implications - By carrying out this research, it has been possible to demonstrate the status of the software development regulations in Santiago de Cali and to propose solutions to the existing risks in the matter, making software developers to self-manage their intellectual property.

Originality - This article is useful for microenterprises and software development companies, as it offers an alternative instrument to protect intellectual property.

Keywords: Software development, intellectual property, copyright, software development contracts.

\footnotetext{
*Profesora investigadora de la Universidad Cooperativa de Colombia (Facultad de Derecho sede Cali), actualmente PhD (C) en Derecho de la Universidad Carlos III de Madrid. Su áreas de investigación son propiedad intelectual, derecho y tecnologías de la información y licenciamiento de software. Email: lainiver@lainiver.com.
}

MUNAR, L. M. El desarrollo de software en Colombia: una perspectiva juridica. The Law, State and Telecommunications Review, Brasilia, v. 10, n. 2, p. 53-66, October 2018. [DOI: https://doi.org/10.26512/lstr.v10i2.21493] 


\section{Resumen}

Propósito - En los últimos años Colombia ha sido un país muy activo en la región apoyando el sector de la innovación y la tecnología, por ende el Ministerio de las tecnologías de la información y las comunicaciones -MINTIC-, ha implementado diversos programas, planes y estrategias que desde el gobierno nacional buscan mejorar la innovación y la tecnología, dentro de la innovación y la tecnología se encuentra el desarrollo de software, también llamado soporte lógico o programa de ordenador 1, que como construcción propia del intelecto humano trate consigo la necesidad de ser protegida por la normatividad de propiedad intelectual, específicamente por las leyes atenientes a Derecho de autor para el caso colombiano. Planes como Vive Digital en Colombia, atienden a la necesidad del país de estar a la vanguardia de la tecnología y buscar la inclusión de la sociedad no solo en el manejo de las tecnologías de la información, sino también en la creación, construcción y desarrollo de esas tecnologías, esto atiende a diversas políticas públicas como son: generación de empleo y reducción de la pobreza.Por lo anterior se hace necesario presentar este artículo como producto del avance de investigación donde se pretende constituir el estado del arte de la legislación sobre software existente en Colombia.

Metodología - Es importante determinar dentro del sistema jurídico colombiano, la reglamentación relevante que regula la propiedad intelectual en relación con la industria que desarrolla software, para ello la investigación que se lleva a cabo es socio ju rídica por cuanto se tendrán en cuenta el crecimiento y consolidación de la industria de desarrollo de software en Colombia, luego se procede a identificar la reglamentación que existe en la materia y a partir de ello determinar el estado de arte en la materia.

Resultados - Se logró condensar el estado del arte en materia jurídica del software como obra dentro de la categoría de Derechos de Autor en Colombia, ya que en la actualidad se encuentran diversos manuales que regulan los derechos de autor de forma general, pero no en materia de software el cual debe ser concebido desde la perspectiva jurídica como un conjunto de productos como: el programa de computador, la descripción de programa y el material auxiliar.

Implicaciones prácticas - Al realizar la presente investigación se logra evidenciar el estado de la reglamentación sobre de desarrollo de software en Santiago de Cali por y se logra proponer soluciones a los riesgos existentes en la materia lo cual trae como consecuencia que la industria desarrolladora de software logre autogestionar su propiedad intelectual.

Originalidad - El presente artículo será útil para las micro y pequeñas empresas desarrolladoras de software. Ya que el estudio realizado que ofrece el presente documento proporcional un instrumento alternativo para proteger su capital más valioso el conocimiento.

Palabras clave: desarrollo de software, propiedad intelectual, derechos de autor, contratos de desarrollo de software.

MUNAR, L. M. El desarrollo de software en Colombia: una perspectiva juridica. The Law, State and Telecommunications Review, Brasilia, v. 10, n. 2, p. 53-66, October 2018. [DOI: https://doi.org/10.26512/lstr.v10i2.21493] 


\section{Introducción}

Alvarez y Restrepo (1997), refieren que la gran ola de conocimientos también conlleva a nuevos objetos de protección y que frente a las diferentes obras protegidas por derechos de autor encontramos el software, el software en la actualidad es una industria que demuestra un desarrollo importante en la materia, para el año 2015 datos del Ministerio de las tecnologías de la información y las comunicaciones de Colombia - MINTIC- señalan que mientras en el 2010 la comercialización de software alcanzaba los 2,6 billones de pesos, 900 millones de dolares aproximadamente, para el 2015 la cifra alcanzó los 7,5 billones de pesos 2,500 millones de dolares. (MINTIC, 2015) "En los últimos años, la industria del software viene en pleno crecimiento, facturando, para el 2012, 4,2 billones de pesos es decir 1,500 millones de dolares aproximadamente en ingresos operacionales, con un crecimiento de 27 por ciento frente al año anterior. Estas cifras muestran el dinamismo que ha tenido el sector en los últimos años, si se compara con el crecimiento de las empresas del resto de la economía, las cuales crecieron entre el 2008 y el 2012 un 7,6 por ciento", destaca la Federación Colombiana de la Industria del Software"2. (Fedesoft,2015).

Según informes del MINTIC las cifras de exportaciones en el sector de las TIC Aumentó durante el año 2014 en un 29\%, cifra que demuestra como Colombia va creciendo en la producción de tecnologías a nivel mundial, también Colombia es reconocido en la región (Suramerica) como el país productor de software con mayor calidad, lo que demuestra la importancia del sector para el crecimiento económico del país y el nivel de experticia que ha conquistado la industria en los últimos años, atendiendo a las tendencias del orden mundial y las demandas sociales del país. En los últimos años el gobierno nacional ha puesto en marcha diferentes programas que impulsan el sector y han consolidado el ecosistema de emprendimientos en la región que si bien, aún no alcanza la cifras en ventas esperado si muestra resultados alentadores para la industria del software. (MINTIC,2015).

Por ende el crecimiento de la industria del software en Colombia es evidente y el Estado Colombiano por medio de programas como Vive Digital ${ }^{3}$ ha realizado aportes importantes en la materia, pues en los últimos años la tecnología ha logrado permear diversos sectores económicos, sociales y académicos, las cifras anteriores muestran como la producción de software es una actividad

\footnotetext{
${ }^{2}$ Disponible en internet en: http://vivircolombia.co/software-100-porciento-colombianosobresale-en-latinoamerica.html, fecha 20 de marzo de 2017.

${ }^{3}$ El Plan Vive Digital en Colombia, tiene objetivos tales como: “(...) Promover el acceso, uso y apropiación de las TIC en el país" Recuperado de internet de: http://www.mintic.gov.co/portal/vivedigital/612/w3-propertyvalue-19436.html, 16 de febrero de 2017.
}

MUNAR, L. M. El desarrollo de software en Colombia: una perspectiva jurídica. The Law, State and Telecommunications Review, Brasilia, v. 10, n. 2, p. 53-66, October 2018. [DOI: https://doi.org/10.26512/lstr.v10i2.21493] 
importante de crecimiento para la economía colombiana y por ende sus prácticas deben gozar de una gestión jurídica integral que permita el sano crecimiento de la industria y la debida ejecución de los contratos.

\section{Marco conceptual de la propiedad intelectual en Colombia}

En Colombia la propiedad intelectual es la protección jurídica que se reconoce a los autores sobre las creaciones que provienen de su ingenio o intelecto, en el campo científico, artístico, literario, industrial y comercial. En términos jurídicos la propiedad intelectual tienen dos regulaciones especiales en la materia, por un lado regula los correspondiente a Derechos de Autor como categoría jurídica que ofrece protección especial a las obras que provienen del intelecto humano pero que pertenecen específicamente al ámbito de lo artístico y literario y la otra categoría jurídica corresponde a la propiedad industrial que abarca todas las creaciones del intelecto humano pero que cumplen una papel en la industria específico como es el caso de las marcas, patentes y diseños industriales. En este sentido, aunque los derechos de autor y la propiedad industrial en principio hacen parte de la propiedad intelectual la regulación jurídica para cada una de ellas goza de una protección especial en cada caso.

En el año 2005, la Corte Constitucional de Colombia, en Sentencia C$1118^{4}$ estableció:

"El objeto que se protege a través del derecho de autor es la obra, esto es “(...) la expresión personal de la inteligencia que desarrolla un pensamiento que se manifiesta bajo una forma perceptible, tiene originalidad o individualidad suficiente, y es apta para ser difundida y reproducida." [3] Dicha protección está condicionada al cumplimiento de los siguientes presupuestos: el derecho de autor protege las creaciones formales no las ideas; la originalidad es condición necesaria para la protección; ella, además, no depende del valor o mérito de la obra, ni de su destino o forma de expresión y, en la mayoría de legislaciones, no está sujeta al cumplimiento de formalidades; cosa distinta es el registro que de ellas lleve el Estado, en el caso colombiano denominado Registro Nacional de Derechos de Autor, el cual tiene fines específicos de publicidad y seguridad jurídica, según se consigna de manera expresa en el artículo 193 de la ley 23 de 1982”.

Los derechos de autor se componen por los derechos patrimoniales y morales, que constituyen un todo para la propiedad sobre las obras que gozan de

\footnotetext{
${ }^{4}$ República de Colombia. Sentencia 1118 de 2005, Corte Constitucional, mediante la cual se realiza un análisis de constitucionalidad al artículo 22 de la Ley 44 de 1993, por establecer una prescripción extintiva cuando el autor no ha ejercido su derecho patrimonial en el transcurso del tiempo.
}

MUNAR, L. M. El desarrollo de software en Colombia: una perspectiva jurídica. The Law, State and Telecommunications Review, Brasilia, v. 10, n. 2, p. 53-66, October 2018. [DOI: https://doi.org/10.26512/lstr.v10i2.21493] 
derechos de autor, sin embargo, en su significado y aplicación cuentan con prerrogativas particulares, como lo estableció la Corte Constitucional colombiana en Sentencia C-837 de 2013 ${ }^{5}$. Los derechos morales versan sobre el derecho a divulgar la obra, el reconocimiento del derecho de paternidad, el derecho a la integridad de la obra y el derecho de retracto, por otro lado se encuentran los derechos patrimoniales que están directamente vinculados con el derecho a reproducir la obra, comunicarla públicamente, venderla, adaptarla y/o cualquier forma de utilización de la obra, por ende en principio es el autor (desarrollador de software DS) quien tiene bajo la esfera de su propiedad la titularidad completa de los derechos patrimoniales y morales de autor sobre su desarrollo.

En materia de derechos de autor, la Ley 23 de 1982, establece las disposiciones generales que regulan la materia de derechos de autor en Colombia, determina en su artículo 1, que los autores de obras literarias, científicas y artísticas gozan de protección en la forma como la ley lo establece y el derecho común. La citada Ley estipula diversas normas respecto a la protección que merecen todas las obras científicas, literarias y artísticas, estableciendo diferentes tipos de derechos de autor como es el caso de derechos de los derechos morales y patrimoniales de autor. En la presente ley no se encuentra definido frontalmente reglamentación en temas de derechos de autor para la industria del software o soporte lógico sinónimos estos en el ámbito jurídico, El soporte lógico es considerado un obra en virtud del Decreto 1360 de 1989 el cual en su artículo 1, reza: "De conformidad con lo previsto en la ley 23 de 1982 sobre Derechos de Autor, el soporte lógico (software) se considera como una creación propia del dominio literario", este sentido se le da la categoría al software como una obra propia del dominio literario y en el artículo 2 de la citada ley se establecen los elementos que lo componen y son: el programa de computador, la descripción del programa y el material auxiliar.

El Decreto 1360 de 1989, marca conceptualmente relevancia jurídica para el software también llamado soporte lógico $^{6}$ en Colombia, ya que más allá de establecer reglamentación para registrar software ante la Dirección Nacional de Derechos de autor, en su artículo 2 establece que el software entendido como soporte lógico comprende uno o varios elementos que son: el programa de

\footnotetext{
${ }^{5}$ República de Colombia. Sentencia 837 de 2013, Corte Constitucional, se establece el análisis de constitucionalidad de los artículos 1, 2, 13, 20, 61, 70, 71, 73, 95-9, 333 y 363 de la Ley 98 de 1993, la cual establece la exención del impuesto sobre las ventas de algunas obras protegidas por los derechos de autor.

${ }^{6}$ Decisión andina 351 de 1993, Articulo 3:“ Expresión de un conjunto de instrucciones mediante palabras, códigos, planes o en cualquier otra forma que, al ser incorporadas en un dispositivo de lectura automatizada, es capaz de hacer que un ordenador -un aparato electrónico o similar capaz de elaborar informaciones -, ejecute determinada tarea u obtenga determinado resultado. El programa de ordenador comprende también la documentación técnica y los manuales de uso"
}

MUNAR, L. M. El desarrollo de software en Colombia: una perspectiva jurídica. The Law, State and Telecommunications Review, Brasilia, v. 10, n. 2, p. 53-66, October 2018. [DOI: https://doi.org/10.26512/lstr.v10i2.21493] 
computador, la descripción de programa y el material auxiliar. Es decir que no se debe concebir el programa de ordenador exclusivamente al conjunto organizado de instrucciones en lenguaje natural o codificado como lo establece el artículo 3 del mencionado Decreto. Sino también se encuentran dos componentes más que en virtud del presente decreto, y eso hace posible el registro del material auxiliar y la descripción del programa, y lograr así la protección jurídica del software a este conjunto de elementos durante toda la vida del autor y 80 años más en el caso de personas naturales y en el caso de personas jurídicas el plazo es de 50 años contados a partir de su divulgación.

Gráfico 1. Concepto de software Decreto 1360 de 1989

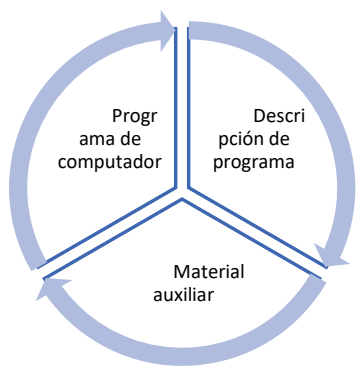

Fuente: Elaboración propia.

Sin embargo, un programa de ordenador puede contar con otros elementos jurídicamente relevantes y que estaría protegidos por el régimen legal de propiedad industrial como es el caso de los diseños gráficos asociados al software, los logos, las marcas y las enseñas comerciales.

De lo anterior cobra relevancia determinar dentro de las empresas desarrolladoras de software la forma como protegen no solo el programa de computador entendido con un conjunto de instrucciones de orden lógico, sino también la descripción de programa entendida en virtud del decreto 1360 como una presentación completa de procedimientos en forma idónea, detallada que logra determinar el conjunto de instrucciones que constituyen el programa de ordenador. Por otro lado, el material auxiliar que es el creado para facilitar su comprensión o aplicación con él es caso de descripción de problemas e instrucciones para el usuario.

MUNAR, L. M. El desarrollo de software en Colombia: una perspectiva juridica. The Law, State and Telecommunications Review, Brasilia, v. 10, n. 2, p. 53-66, October 2018. [DOI: https://doi.org/10.26512/lstr.v10i2.21493] 
De acuerdo a las entrevistas no estructuradas realizadas a 5 microempresas desarrolladoras de software del Departamento del Valle del Cauca, la selección de dichas empresas se realizó en el mes de junio del año $2017^{7}$, de manera aleatoria a partir de la base de datos de la Cámara de Comercio de Cali año 2015, teniendo en cuenta como criterios de selección que las empresas estuvieran legalmente constituidas y con registro mercantil vigente, además que su objeto social principal fuera el descrito en el código CIIU 6201 ; actividades de desarrollo de sistemas informáticos ${ }^{8}$, donde se determinó que estás empresas al realizar de ventas de servicios de desarrollo de software o de programas de ordenador, generalmente entregan a sus clientes una propuesta técnica, las propuestas técnicas analizadas en virtud de la presente investigación contenían:

1. Descripción del programa: incluyen en este ítem, funcionalidades, módulos, tecnología necesaria para su funcionamiento, puede incluir diseños o representaciones gráficas o textuales de funcionamiento del sistema.

2. Valor o precio de la propuesta

3. Utilidad del software para solucionar un problema o mejorar una situación determinada a sus clientes.

Seguido a la anterior los contratos realizados por las compañías entrevistadas, son específicamente contratos de licenciamiento, donde se definen un usuario licenciatario (cliente) y un proveedor licenciante (empresa desarrolladora). En los contratos establecidos no se encuentra una protección frontal a los tres componentes del software que determina el Decreto 1360 de 1989 en su artículo tercero. Por otro lado, no se evidencian en las compañías la cesión de derechos por parte de los desarrolladores a las compañías distribuidoras de software. ${ }^{9}$

\footnotetext{
${ }^{7}$ La aplicación de estas entrevistas se realizó dentro del marco de la investigación " Gestión jurídica de la propiedad intelectual en Mypimes desarrolladoras de software" de la Facultad de Derecho de la Universidad Cooperativa de Colombia Sede Cali desarrollada durante los años 2017 y 2018, donde participó como investigadora principal: Phd (C) Lainiver Mendoza Munar, como coinvestigadores Mg. Josefina de Llano Feliu y de la Universidad de La Laguna de Santa Cruz de Tenerife (España) el PhD Luis Capote Perez, en el virtud de la mencionada investigación se desarrolló la monografía de grado denominada: "Diagnóstico de las prácticas de autogestión para la protección de la propiedad intelectual en empresas Pymes de desarrollo de software en el Valle del Cauca" cuya directora fue la coinvestigadora profesora investigadora Josefina del Llano Feliu y cuyo autor es Jair Garcia Mejia.

${ }^{8}$ El código CIIU (Clasificación Industrial Internacional Uniforme) es la clasificación de actividades económicas de los empresarios que utiliza la Dirección de Impuestos y Aduanas Nacionales (DIAN) en Colombia.
}

MUNAR, L. M. El desarrollo de software en Colombia: una perspectiva jurídica. The Law, State and Telecommunications Review, Brasilia, v. 10, n. 2, p. 53-66, October 2018. [DOI: https://doi.org/10.26512/lstr.v10i2.21493] 
Existe en términos generales un desconocimiento a la distinción en los conceptos de derechos de autor y propiedad industrial y los regímenes de protección que ofrecen y que son necesario para gestionar su propiedad intelectual. Determinando lo anterior es importante tener en cuenta la variable "gestión de la propiedad intelectual" para la elaboración de instrumentos para aplicación futura, que proveen la posibilidad de realizar un diagnóstico a un mayor número de empresas desarrolladoras de software de la ciudad y que tenga en cuenta indicadores como: redacción de contratos, tipos de contratos utilizados en virtud de la transacciones comerciales donde el objeto contractual es software, protección de datos personales, propiedad sobre el software, confidencialidad, objetos materia de protección por el régimen de propiedad industrial.

En virtud de la anterior, es oportuno determinar que sujetos y obras protege y que derechos reconoce tanto el régimen legal de derechos de autor con el de propiedad industrial en Colombia, establecidos en la Tabla 1.

Tabla 1 - Derechos de Autor y Propiedad Industrial

\begin{tabular}{|c|c|c|}
\hline Items & Derechos de autor & Propiedad industrial \\
\hline $\begin{array}{l}\text { Sujetos } \\
\text { que } \\
\text { protege }\end{array}$ & $\begin{array}{l}\text { Autores de obras artísticas, } \\
\text { científicas y literarias. (Ley } \\
23 \text { de 1982) }\end{array}$ & $\begin{array}{l}\text { Inventores, diseñadores, } \\
\text { productores, extractores, } \\
\text { fabricantes, artesanos } \\
\text { siempre y cuanto sus } \\
\text { creaciones tengan } \\
\text { aplicación industrial. } \\
\text { (Decisión } 486 \text { de la } \\
\text { Comunidad Andina de } \\
\text { Naciones). }\end{array}$ \\
\hline $\begin{array}{l}\text { Obras } \\
\text { que } \\
\text { protege }\end{array}$ & $\begin{array}{l}\text { Libros, Folletos, } \\
\text { conferencias, alocuciones, } \\
\text { sermones, obras dramáticas, } \\
\text { dramático-musicales, } \\
\text { coreográficas, pantomimas, } \\
\text { composiciones musicales } \\
\text { con o sin letras, obras } \\
\text { cinematográficas, } \\
\text { videogramas, las obras de } \\
\text { dibujo, puntura. } \\
\text { Arquitectura, escultura. } \\
\text { Grabado, litografía, obras } \\
\text { de arte aplicado, } \\
\text { ilustraciones, mapas, } \\
\text { planos, croquis, obras } \\
\text { plásticas de geografía, } \\
\text { topografía, arquitectura o } \\
\text { las ciencias. (Ley } 23 \text { de } \\
\text { 1982) }\end{array}$ & $\begin{array}{l}\text { Patentes de invención, } \\
\text { patentes modelo de utilidad, } \\
\text { esquemas de trazado de } \\
\text { circuitos integrados, diseño } \\
\text { industrial, marcas, lemas } \\
\text { comerciales, marcas de } \\
\text { certificación, nombre } \\
\text { comercial, rótulos o } \\
\text { enseñas, denominaciones } \\
\text { de origen, indicaciones de } \\
\text { procedencia, entre otros. } \\
\text { (Decisión } 486 \text { de la } \\
\text { Comunidad Andina de } \\
\text { Naciones). }\end{array}$ \\
\hline
\end{tabular}

MUNAR, L. M. El desarrollo de software en Colombia: una perspectiva juridica. The Law, State and Telecommunications Review, Brasilia, v. 10, n. 2, p. 53-66, October 2018. [DOI: https://doi.org/10.26512/lstr.v10i2.21493] 


\begin{tabular}{|l|l|l|}
\hline $\begin{array}{l}\text { Derechos } \\
\text { que } \\
\text { otorga al } \\
\text { autor } \\
\text { y/o } \\
\text { creador }\end{array}$ & $\begin{array}{l}\text { 1. Derechos patrimoniales } \\
\text { de autor }\end{array}$ & $\begin{array}{l}\text { 2. Derechos morales de } \\
\text { autor }\end{array}$ \\
\hline
\end{tabular}

\section{Marco normativo de la propiedad intelectual en Colombia}

Cabe resaltar que, en Colombia, la ley marco sobre propiedad intelectual es la ley 23 de 1982. Esta a su vez ha sufrido diversos cambios y actualizaciones jurídicas a través del tiempo. Una de las actualizaciones más importantes es la que introdujo el Decreto 1360 de 1989 donde se establece que el software es una creación propia del dominio humano y por tal disposición debe considerar como una obra del dominio literario. Sin embargo, en Colombia se evidencia diversa reglamentación que regula los derechos de autor, en algunos casos la norma regula de manera frontal aspectos del software y en otros casos al regular derechos de autor de manera genérica lo que inherentemente impacta el software como bien intangible y su regulación, dentro de la normatividad entendiéndose como Leyes y Decretos, en Colombia se encuentra la siguiente normatividad:

Tabla 2 - Regulación del software en Colombia

\begin{tabular}{|c|c|}
\hline Norma & Aspecto que regula \\
\hline $\begin{array}{l}\text { Ley } 29 \text { de } 1944 \text { (diciembre } \\
\text { 15) }\end{array}$ & $\begin{array}{l}\text { Por la cual se dictan disposiciones sobre prensa, } \\
\text { Artículo } 8^{\circ} \text {.- Todo cartel o volante tendrá que estar } \\
\text { debidamente firmado por su autor. }\end{array}$ \\
\hline $\begin{array}{l}\text { LEY } 23 \text { DE } 1982 \\
\text { (enero 28) }\end{array}$ & Ley general sobre derechos de autor. \\
\hline $\begin{array}{l}\text { LEY } 33 \text { DE } 1987 \text { (octubre } \\
\text { 26) }\end{array}$ & $\begin{array}{l}\text { "por medio de la cual se aprueba el 'Convenio de } \\
\text { Berna para la protección de las obras literarias y } \\
\text { artísticas', del } 9 \text { de septiembre de } 1886 \text {, } \\
\text { completado en París el } 4 \text { de mayo de } 1896 \text {, } \\
\text { revisado en Berlín el } 13 \text { de noviembre de } 1908 \text {, } \\
\text { completado en Berna el } 20 \text { de marzo de } 1914 \text { y } \\
\text { revisado en Roma el } 2 \text { de junio de } 1928 \text {, en } \\
\text { Bruselas el } 26 \text { de junio de } 1948 \text {, en Estocolmo el } \\
14 \text { de julio de } 1967 \text { y en París el } 24 \text { de julio de } \\
1971 \text { " }\end{array}$ \\
\hline $\begin{array}{l}\text { LEY } 1360 \text { DE } 1989 \text { (junio } \\
\text { 23) }\end{array}$ & $\begin{array}{l}\text { "Por el cual se reglamenta la inscripción de soporte } \\
\text { lógico (software) en el Registro Nacional del } \\
\text { Derecho de Autor" y donde en su artículo } 3 \text { se } \\
\text { establece el soporte lógico como una obra distinta } \\
\text { a la obra literaria. }\end{array}$ \\
\hline $\begin{array}{l}\text { Decreto } 162 \text { de } 1996 \text { (enero } \\
\text { 22) }\end{array}$ & $\begin{array}{l}\text { Por el cual se reglamenta Decisión Andina } 351 \text { de } \\
1993 \text { la Régimen común sobre derecho de autor y } \\
\text { derechos conexos, para la Comunidad Andina de }\end{array}$ \\
\hline
\end{tabular}

MUNAR, L. M. El desarrollo de software en Colombia: una perspectiva juridica. The Law, State and Telecommunications Review, Brasilia, v. 10, n. 2, p. 53-66, October 2018. [DOI: https://doi.org/10.26512/lstr.v10i2.21493] 


\begin{tabular}{|c|c|}
\hline & $\begin{array}{l}\text { Naciones, integrada por Bolivia, Colombia, } \\
\text { Ecuador y Perú. }\end{array}$ \\
\hline $\begin{array}{l}\text { Ley } 44 \text { de } 1993 \\
\text { (febrero 5) }\end{array}$ & $\begin{array}{l}\text { Por la cual se modifica y adiciona la Ley } 23 \text { de } \\
1982 \text { y se modifica la Ley } 29 \text { de } 1944 \text {, se } \\
\text { establecen las disposiciones para el registro } \\
\text { Nacional del Derecho de Autor. }\end{array}$ \\
\hline $\begin{array}{l}\text { Decreto } 1474 \text { de } 2002 \text { (julio } \\
\text { 15) }\end{array}$ & $\begin{array}{l}\text { "Por el cual se promulga el "Tratado de la OMPI, } \\
\text { Organización Mundial de la Propiedad Intelectual, } \\
\text { sobre Derechos de Autor (WCT)", adoptado en } \\
\text { Ginebra, el veinte (20) de diciembre de mil } \\
\text { novecientos noventa y seis (1996)". }\end{array}$ \\
\hline $\begin{array}{l}\text { Ley } 599 \text { de } 2000 \\
\text { (julio 24) }\end{array}$ & $\begin{array}{l}\text { En el título VIII articulo } 270 \text { al } 272 \text { se establecen } \\
\text { los delitos de contra la protección de la } \\
\text { información y de los datos, los delitos establecidos } \\
\text { como vulneración a los derechos de autor son: } \\
\text { violación a los derechos morales de autor, } \\
\text { defraudación a los derechos patrimoniales de autor } \\
\text { y violación a los mecanismos de protección de los } \\
\text { derechos patrimoniales de autor y otras } \\
\text { defraudaciones. }\end{array}$ \\
\hline $\begin{array}{l}\text { Ley } 1273 \text { de } 2009 \\
\text { (enero 05) }\end{array}$ & $\begin{array}{l}\text { Por medio de esta Ley se modifica el Código Penal } \\
\text { y se crea un nuevo bien jurídico tutelado en } \\
\text { Colombia, denominado "De la protección de la } \\
\text { información y de los datos", al mismo tiempo se } \\
\text { protegen los sistemas que utilicen las tecnologías } \\
\text { de la información y las comunicaciones. }\end{array}$ \\
\hline $\begin{array}{l}\text { Ley } 1341 \text { de } 2009 \\
\text { (julio 29) }\end{array}$ & $\begin{array}{l}\text { "Por la cual se definen Principios y conceptos } \\
\text { sobre la sociedad de la información y la } \\
\text { organización de las Tecnologías de Información y } \\
\text { las Comunicaciones -TIC, se crea la Agencia } \\
\text { Nacional del Espectro y se dictan otras } \\
\text { disposiciones" compiló los Decretos } \\
\text { Reglamentarios números } 1360 \text { de } 1989 \text { y } 460 \text { de } \\
1995 \text { (Inscripción de soporte lógico (software) y el } \\
\text { Registro Nacional del Derecho de Autor, } \\
\text { respectivamente), } 3942 \text { de } 2010 \text { y } 1258 \text { de } 2012 \\
\text { (Gestión Colectiva del Derecho de Autor y } \\
\text { facultades de Inspección, Vigilancia y Control de } \\
\text { la Dirección Nacional de Derecho de Autor, } \\
\text { respectivamente). }\end{array}$ \\
\hline LEY 1450 de 2011 & $\begin{array}{l}\text { Por la cual se expide el Plan Nacional de } \\
\text { Desarrollo, 2010-2014. }\end{array}$ \\
\hline LEY 1520 DE 2012 & $\begin{array}{l}\text { Por medio de la cual se implementan compromisos } \\
\text { adquiridos por virtud del "Acuerdo de Promoción } \\
\text { Comercial", suscrito entre la República de } \\
\text { Colombia y los Estados Unidos de América y su } \\
\text { "Protocolo Modificatorio, en el Marco de la } \\
\text { Política de Comercio Exterior e Integración } \\
\text { Económica". }\end{array}$ \\
\hline $\begin{array}{l}\text { El Decreto Reglamentario } \\
\text { Único del Sector Interior } \\
\text { No. } 1066 \text { de } 2015\end{array}$ & $\begin{array}{l}\text { En el capítulo 3, artículo } 2,6,1,3,1 \text { y SS. Se definen } \\
\text { los elementos del software, se establecen los } \\
\text { requisitos para la inscripción del soporte lógico, de } \\
\text { acuerdo a lo contenido en el Decreto } 1360 \text { de } 1989 \text {. }\end{array}$ \\
\hline
\end{tabular}

MUNAR, L. M. El desarrollo de software en Colombia: una perspectiva jurídica. The Law, State and Telecommunications Review, Brasilia, v. 10, n. 2, p. 53-66, October 2018. [DOI: https://doi.org/10.26512/lstr.v10i2.21493] 
FUENTE: Dirección Nacional de Derecho de Autor de Colombia ${ }^{10}$

De acuerdo con lo anterior, es posible observar que, en materia de producción normativa en la categoría de leyes en Colombia, encontramos la siguiente producción:

Gráfico 2 - Producción legislativa sobre derechos de autor relevante para el desarrollo de software en Colombia

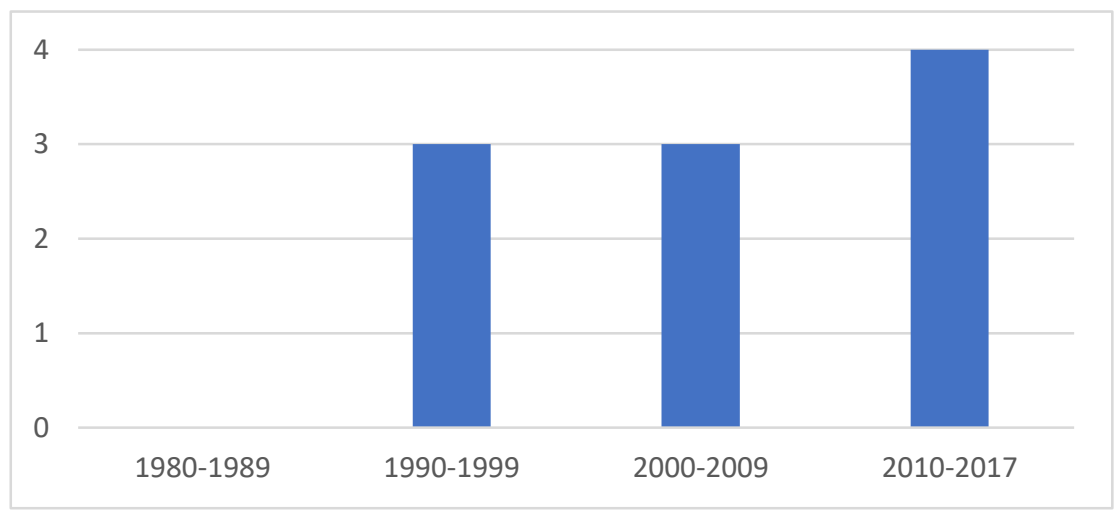

FUENTE: Dirección Nacional de Derecho de Autor de Colombia. ${ }^{11}$

Se evidencia una producción legislativa similar en los la última década de los años noventa y la primera del segundo milenio, y un incremento en la materia después del año 2010. Donde además también se encuentran un fuerte incremento en diversos programas del Estado que buscan apalancar la creación de un ecosistema digital.

\section{Presunciones de derecho que operan para el desarrollo del software en Colombia}

Según la Corte Constitucional de Colombia en Sentencia C-731 de 2005: "la palabra presumir viene del término latino "praesumere" que significa "tomar antes, porque por la presunción toma o tiene por cierto un hecho, un derecho o una voluntad, antes de que la voluntad, el derecho o el hecho se prueben". En este sentido la Sentencia C-238 de 1999 define que la existencia de las presunciones son de índole probatoria de un supuestos de hecho establecidos, en este sentido las presunciones legales "iuris tantum" admiten prueba en contrario y son las que

\footnotetext{
${ }^{10}$ Dirección Nacional de Derecho de Autor de Colombia,disponible en internet en: http://derechodeautor.gov.co/web/guest/leyes, 24 de agosto de 2018.

${ }^{11}$ Dirección Nacional de Derecho de Autor de Colombia,disponible en internet en: http://derechodeautor.gov.co/web/guest/leyes, 24 de agosto de 2018.
} 
se encuentran definidas en una norma jurídica y únicamente para probar un determinado suceso a la parte que favorece la presunción basta con demostrar que los hechos se verificaron dentro de lo establecido por la ley, corresponde por ende a la parte contraria demostrar la inexistencia del hecho que se presume. Algunas presunciones de legales relevantes que se encuentran en la legislación sobre derechos de autor, en la materia son:

a. Obra inédita: En el artículo 4 del Decreto 1360 de 1989, se establece que el software será considerado como obra inédita, a menos que su titular manifieste que no lo es.

b. Propiedad intelectual de obras en cumplimiento de un contrato de prestación de servicios o un contrato de trabajo: en las obras creadas bajo un contrato de prestación de servicios o un contrato de trabajo: Se establece en el artículo 28 se presume que los derechos patrimoniales han sido transferidos al encargante o empleador, es requisito en este caso que le contrato conste por escrito para que la presunción opere.

c. Derechos patrimoniales de autor: Artículo 30 de la ley 1450 de 2011 A falta de mención del tiempo de la transferencia de derechos de patrimoniales de autor en un contrato escrito se limitará la transferencia a 5 años y toda transferencia debe constar por escrito.

d. Exclusividad: Artículo 30 de la ley 1450 de 2011 todos los actos o contratos que impliquen exclusividad deberán ser inscritos en el Registro Nacional del Derecho de Autor, para efectos de publicidad y oponibilidad ante terceros.

Estas cuatro presunciones que operan legislativamente en materia de derechos de autor y desarrollo de software, tienen una gran relevancia para la industria y se hace necesario su comunicación y aplicación en los diferentes contratos que se elaboren al interior de ellas y así fortalecer la gestión de la propiedad intelectual en su relación con los clientes y lograr la ejecución de negocios jurídicamente estables.

\section{Conclusiones}

Por lo que determina el Decreto 1360 de 1989, es importante revisar cómo se compone el software y de acuerdo a lo anterior que elementos de cada una de sus etapas de desarrollo son objeto de protección bajo registro ante la Dirección Nacional de Derechos de Autor de Colombia, teniendo en cuenta: el programa de ordenador, la descripción del programa y el material auxiliar.

El documento escrito es uno de los elementos más importantes a tener en cuenta para la elaboración de un contrato de desarrollo de software, pues existen

MUNAR, L. M. El desarrollo de software en Colombia: una perspectiva jurídica. The Law, State and Telecommunications Review, Brasilia, v. 10, n. 2, p. 53-66, October 2018. [DOI: https://doi.org/10.26512/lstr.v10i2.21493] 
presunciones jurídicas diversas que pueden ser contrarias a la voluntad de las partes y el elemento escrito puede regularlas conforme al consentimiento de los contratante; de esta forma las partes realmente pueden plasmar su voluntad y probarla, de lo contrario operaran las presunciones establecidas por la ley, las cuales pueden generar consecuencias jurídicas distintas a las deseadas por las obligados en virtud de un contrato.

Frente a los programas de ordenador y los derechos de autor en Colombia, se puede encontrar la ley 1450 de 2011, la cual en su artículo 28, introdujo a la Ley 23 de 1982 un cambio normativo donde se establece básicamente que aún en un contrato laboral o de prestación de servicios; el autor es el titular originario de los derechos patrimoniales y morales de autor, salvo pacto en contrario donde se establece que los derechos patrimoniales de la obra han sido cedidos al empleador, es decir que se crea un presunción legal que requiere especial atención para las empresas que desarrollan programas informáticos.

La reglamentación existente en la materia es escasa, sin embargo, para efectos de elaboración de contratos, se marcan unas reglas generales relevantes al momento de su elaboración, lo que es importante tener en cuenta en la industria, especialmente para los microempresarios y emprendedores que pueden llegar a omitir la realización de contratos escritos por el desconocimiento de estas reglas.

Conforme a lo anterior para continuar con el desarrollo de la investigación, es indispensable la elaboración de instrumentos que se aplicarán a las empresas de desarrollo de software y establecer los aspectos más relevantes de la legislación existente en materia, además se deben tener en cuenta las presunciones en derecho y como operan, para definir de acuerdo a los resultados arrojados, el conocimiento o desconocimiento en la elaboración de contratos por parte de las empresas de desarrollo de software, tomando las listas de empresas desarrolladoras de Santiago de Cali, Valle Colombia.

El contrato de desarrollo de software requiere una especial atención para su elaboración, precisamente porque no cuenta con estandarización normativa en Colombia, lo cual lo define como un contrato eminente atípico y más allá de la elaboración de contratos se requiere conocer los elementos jurídicos del software, revisando la legislación existente y como se conjuga para materializar la regulación a nivel contractual especial en la materia.

\section{Referencias}

LIPSZYC, D., Villalba, C., Uchtenhagen, Ulrich. La protección del derecho de autor en el Sistema interamericano, Editorial Universidad del Externado, Bogotá, Colombia, 1998.

MUNAR, L. M. El desarrollo de software en Colombia: una perspectiva juridica. The Law, State and Telecommunications Review, Brasilia, v. 10, n. 2, p. 53-66, October 2018. [DOI: https://doi.org/10.26512/lstr.v10i2.21493] 
ALVAREZ, M., Restrepo, Luz. El derecho de autor y software. Medellín, Colombia, Editorial Universidad Pontificia Bolivariana, 1997.

ARRUÑADA, B. Teoría Contractual de la empresa. Editorial Marcial Pons, 1998.

BYGRAVE, L. A. Data privacy law an international perspectiva. Londres: Oxford University Press, 2014.

BUITRAGO, L. E. Derecho intelectual. Editorial librería ediciones del profesional, Segunda Edición, 2003.

DE LUCCA, N.Contratación informática y telemática. Pontificia Universidad Javeriana, Bogotá, Colombia, 2012.

GHERSI, C. A. Metodología de la investigación en las ciencias jurídicas. Editorial GOWA Ediciones profesionales, Buenos Aires, Argentina, 2007.

GIRALDO A. J. Metodología y técnica de la investigación socio jurídica, Bogotá, Colombia, 2012.

Grupo de Estudios en internet. Contenido electrónico, telecomunicaciones e informática. GECT. Derecho \& TIC 10.0. Universidad de los Andes, Bogotá, Colombia, 2011.

LOPEZ ROMÁN L., Montenegro de Timarán M. I., Tapia Fierro R. M. La investigación, eje fundamental en la enseñanza del derecho, guía práctica, Editorial Universidad Cooperativa de Colombia. Bogotá, Colombia,2006.

PEÑA, V. D. BAZZANI, J. D. Los aspectos legales de la legislación en la nube. Universidad del Externado de Colombia, Bogotá, Colombia, 2010

PRESSMAN, R.S. Ingeniería del software, un enfoque práctico, séptima edición. Editorial McGraw Hill, Mexico, 2010.

REYES,V. F. derecho societario en estados unidos y la unión europea. Editorial Legis, Bogotá, Colombia, 2013.

República de Colombia. Sentencia 1011, Corte Constitucional, 2008.

República de Colombia. Sentencia C-748. Corte Constitucional, 2011.

MUNAR, L. M. El desarrollo de software en Colombia: una perspectiva juridica. The Law, State and Telecommunications Review, Brasilia, v. 10, n. 2, p. 53-66, October 2018. [DOI: https://doi.org/10.26512/lstr.v10i2.21493] 